\title{
Optimal Perturbation Iteration Method for Solving Telegraph Equations
}

\author{
Necdet Bildik* \\ 45140, Manisa, Turkey. \\ *Corresponding author. Tel.: +(90)0236 201 3203; email: necdet.bildik@cbu.edu.tr \\ Manuscript submitted March 28, 2017; accepted May 15, 2017. \\ doi: 10.17706/ijapm.2017.7.3.165-172
}

Manisa Celal Bayar University, Faculty of Arts and Science, Department of Mathematics, Muradiye Campus,

\begin{abstract}
In this work, we obtain the approximate solutions for the telegraph equations by using optimal perturbation iteration technique. We consider two examples to illustrate the proposed method. The present paper also unveils that optimal perturbation iteration technique converges fast to the accurate solutions of the given equations at lower order of iterations.
\end{abstract}

Key words: Optimal perturbation iteration method, telegraph equation.

\section{Introduction}

Many scientific and technological problems in natural and engineering sciences are described by linear and nonlinear differential equations, mostly by partial differential equations. For instance, the heat flow and the wave propagation phenomena are modeled by nonlinear partial differential equations in physics and applied mathematics. The dispersion of a chemically reactive material is also defined by linear and nonlinear partial differential equations. Additionally, most physical phenomena of fluid dynamics, electricity, plasma physics, quantum mechanics, and propagation of shallow water waves with many other models are conducted by these types of differential equations. Therefore, a substantial amount of work has been scrutinized for solving such models [1]-[12].

Many realistic partial differential equations are nonlinear and most of them do not have exact analytic solutions, so numerical methods are needed to handle with them easily. Latterly, several techniques have been used for the solutions of such problems [13]-[15]. If there are exact solutions, these numerical methods often give approximate solutions that quickly converge to the correct solutions. The search for exact or approximate solutions of these partial differential equations will assist us to better understand the phenomena behind these models.

The telegraph equation is one of the most important partial differential equations that define the wave propagation of electrical signals in a cable transmission line. Many researchers have used various numerical and analytical methods to solve the telegraph equation [16]-[18]. The standard form of the telegraph equation can be given as:

$$
u_{x x}=a u_{t t}+b u_{t}+c u
$$

where $u(x, t)$ may be voltage or current through the wire at position $x$ and time $t$. And also $a=L C$, 
$b=G L+R C$ and $c=G R$ where $G$ is conductance of resistor, $R$ is resistance of resistor, $L$ is inductance of coil and $C$ is capacitance of capacitor [18].

In this research, we solve two types of the telegraph equation by using optimal perturbation iteration method (OPIM). We also give some figures that prove the accuracy of the new technique.

\section{OPIM for the Telegraph Equation}

Recently, perturbation iteration method and optimal perturbation iteration method (OPIM) have been effectively used to cope with the strongly nonlinear differential equations [19]-[24]. We now try to design OPIM for the Telegraph equations as follows:

a) Take the given partial differential equation as:

$$
F\left(u_{x x}, u_{t t}, u_{t}, u, \varepsilon\right)=0
$$

where $u=u(x, t)$ and $\varepsilon$ is the perturbation parameter can be embedded into the Eq. 2 as:

$$
F=u_{x x}-a u_{t t}-b u_{t}-\varepsilon c u
$$

To make calculations easier, $F$ can be split as:

$$
F=S+R
$$

where $S$ is the simpler part, which can be easily managed and $R$ is the remaining part which is principal for OPIM. For the Eq. (2), $R$ and $S$ can be taken as:

$$
\begin{aligned}
& R\left(u_{t t}, u, \varepsilon\right)=-a u_{t t}-\varepsilon c u \\
& S\left(u_{x x}, u_{t}\right)=u_{x x}-b u_{t}
\end{aligned}
$$

b) The approximate solution with one correction term in the perturbation expansion

$$
u_{n+1}=u_{n}+\varepsilon\left(u_{c}\right)_{n}
$$

is taken where $\left(u_{c}\right)_{n}$ is the $n$th correction term. To get an algorithm, we substitute (6) into (3) then expand it in a series at $\varepsilon=0$ with first derivative gives:

$$
F+F_{u_{t t}}\left(\left(u_{c}\right)_{n}\right)_{t t} \varepsilon+F_{u_{x x}}\left(\left(u_{c}\right)_{n}\right)_{x x} \varepsilon+F_{u_{t}}\left(\left(u_{c}\right)_{n}\right)_{t} \varepsilon+F_{u}\left(u_{c}\right)_{n} \varepsilon+F_{\varepsilon} \varepsilon=0
$$

or

$$
R+R_{u_{t t}}\left(\left(u_{c}\right)_{n}\right)_{t t} \varepsilon+R_{u_{x x}}\left(\left(u_{c}\right)_{n}\right)_{x x} \varepsilon+R_{u_{t}}\left(\left(u_{c}\right)_{n}\right)_{t} \varepsilon+R_{u}\left(u_{c}\right)_{n} \varepsilon+R_{\varepsilon} \varepsilon=-S
$$

where 


$$
R_{u}=\frac{\partial R}{\partial u}, R_{\varepsilon}=\frac{\partial R}{\partial \varepsilon}, R_{u_{t t}}=\frac{\partial R}{\partial u_{t t}}, R_{u_{x x}}=\frac{\partial R}{\partial u_{x x}}
$$

If these calculations are done for the equation (3), one has

$$
a\left(\left(u_{c}\right)_{n}\right)_{t t}=\left(u_{n}\right)_{x x}-a\left(u_{n}\right)_{t t}-b\left(u_{n}\right)_{t}-c u_{n}
$$

which is called the optimal perturbation iteration algorithm (OPIA) for the telegraph equation (1) . To trigger the iterations, a trial function $u_{0}$ is picked sternly according to the initial conditions. After that the first correction term $\left(u_{c}\right)_{0}$ can be figured out from the algorithm (10) via $u_{0}$.

c) To increase the effectiveness of method, we use the following equation:

$$
u_{n+1}=u_{n}+P_{n}\left(u_{c}\right)_{n}
$$

where $P_{0}, P_{1}, P_{2}, \ldots$ are convergence control parameters which enables us to adjust the convergence.

Advancing for $n=0,1, \ldots$, more approximate solutions are reached as:

$$
\begin{aligned}
& u_{1}=u\left(x, t ; P_{0}\right)=u_{0}+P_{0}\left(u_{c}\right)_{0} \\
& u_{2}\left(x, t ; P_{0}, P_{1}\right)=u_{1}+P_{1}\left(u_{c}\right)_{1} \\
& \quad \vdots \\
& u_{m}\left(x, t ; P_{0}, \ldots, P_{m-1}\right)=u_{m-1}+P_{m-1}\left(u_{c}\right)_{m-1}
\end{aligned}
$$

Substituting $u_{m}$ into the Eq. (1), the general problem will become:

$$
\operatorname{Re}\left(x, t ; P_{0}, \ldots, P_{m-1}\right)=F\left(\left(u_{m}\right)_{x x},\left(u_{m}\right)_{t t},\left(u_{m}\right)_{t}, u_{m}\right)
$$

or

$$
\operatorname{Re}\left(x, t ; P_{0}, \ldots, P_{m-1}\right)=R\left(u_{m}\left(x, t ; P_{0}, \ldots, P_{m-1}\right)\right)+S\left(u_{m}\left(x, t ; P_{0}, \ldots, P_{m-1}\right)\right) .
$$

Seemingly, if $\operatorname{Re}\left(x, t ; P_{0}, \ldots, P_{m-1}\right)=0$ then the approximation $u_{m}\left(x, t ; P_{0}, \ldots, P_{m-1}\right)$ will be the accurate solution. However, such case will not often arise for nonlinear equations, but one can shrink the functional

$$
J\left(P_{0}, \ldots, P_{m-1}\right)=\int_{0}^{T} \int_{a}^{b} \operatorname{Re}^{2}\left(x, t ; P_{0}, \ldots, P_{m-1}\right) d x d t
$$

where $a, b$ and $T$ are elected from the domain of the problem. $P_{0}, P_{1}, \ldots$ can be optimally obtained 
from

$$
\frac{\partial J}{\partial P_{0}}=\frac{\partial J}{\partial P_{1}}=\ldots=\frac{\partial J}{\partial P_{m-1}}=0
$$

The approximate solution of order $m$ can be defined after substituting $P_{0}, P_{1}, \ldots$ into the last one of the Eqs. (12). For more data about OPIM, please see [19]-[24].

\section{Examples}

In this section, we give some illustrations to show the efficiency of the presented method. The accuracy of OPIM is assessed by comparison with the known exact solutions.

Example 1) Consider the Telegraph equation [18],

under the initial conditions

$$
u_{t t}+u_{t}+u=u_{x x}
$$

$$
u(x, 0)=e^{x}, \quad u_{t}(x, 0)=-e^{x} \quad(0<x \leq 1, t \geq 0)
$$

which has an exact solution $u(x, t)=e^{x-t}$.

Trial function $u_{0}$ can be selected as

$$
u_{0}=e^{x}
$$

Using the algorithm (10) and the Eq. (19) with initial conditions, first correction term is obtained:

$$
\left(u_{c}\right)_{0}=-t e^{x}
$$

Therefore, the OPIM solution of first order is:

$$
u_{1}=e^{x}-P_{0} t e^{x}
$$

Performing the same calculations, one can get

$$
\begin{gathered}
u_{2}=\mathrm{e}^{x}-\mathrm{e}^{x} t P_{0}+\frac{1}{2} \mathrm{e}^{x} t\left(-2+t P_{0}\right) P_{1} \\
u_{3}=\mathrm{e}^{x}-\mathrm{e}^{x} t P_{0}+\frac{1}{2} \mathrm{e}^{x} t\left(-2+t P_{0}\right) P_{1}-\frac{1}{6} \mathrm{e}^{x} t\left(6-3 t P_{0}-3 t P_{1}+3 t P_{0} P_{1}+t^{2} P_{0} P_{1}\right) P_{2}
\end{gathered}
$$

To determine $P_{0}, P_{1}, P_{2}$, we form the following residual: 


$$
\begin{aligned}
& \operatorname{Re}\left(x, t ; P_{0}, P_{1}, P_{2}\right)=\left(u_{3}\right)_{x x}-\left(u_{3}\right)_{t t}-\left(u_{3}\right)_{t}-u=\mathrm{e}^{x} P_{0}-\mathrm{e}^{x} P_{0} P_{1} \\
& -\frac{1}{2} \mathrm{e}^{x} t P_{0} P_{1}-\frac{1}{2} \mathrm{e}^{x}\left(-2+t P_{0}\right) P_{1}+\frac{1}{3} \mathrm{e}^{x} t P_{0} P_{1} P_{2}+\frac{1}{3} \mathrm{e}^{x}\left(-3 P_{0}-3 P_{1}+3 P_{0} P_{1}+2 t P_{0} P_{1}\right) P_{2} \\
& +\frac{1}{6} \mathrm{e}^{x} t\left(-3 P_{0}-3 P_{1}+3 P_{0} P_{1}+2 t P_{0} P_{1}\right) P_{2}+\frac{1}{6} \mathrm{e}^{x}\left(6-3 t P_{0}-3 t P_{1}+3 t P_{0} P_{1}+t^{2} P_{0} P_{1}\right) P_{2}
\end{aligned}
$$

for the third order approximation. For $a=0, b=1$ and $T=1$ the Eq. (15) changes to

$$
J\left(P_{0}, P_{1}, P_{2}\right)=\int_{0}^{1} \int_{0}^{1} R e^{2}\left(x, t ; P_{0}, P_{1}, P_{2}\right) d x d t
$$

By solving

$$
\frac{\partial J}{\partial P_{0}}=\frac{\partial J}{\partial P_{1}}=\frac{\partial J}{\partial P_{2}}=0
$$

one can have

$$
P_{0}=0.9023456, P_{1}=-0.0120563, P_{2}=0.1031044
$$

Substituting these constants into the Eq. (23), approximate OPIM solution of third order arises as:

$$
\begin{aligned}
& u_{3}=\mathrm{e}^{x}-0.902345 \mathrm{e}^{x} t-0.006028 \mathrm{e}^{x}(-2+0.902345 t) t- \\
& 0.01718399999 \mathrm{e}^{x} t\left(6-2.703503013 t-0.010878671 t^{2}\right)
\end{aligned} .
$$

If the iteration continues, approximate results can be found in higher order. Fig. 1 gives absolute errors for approximate results in the 6th order.

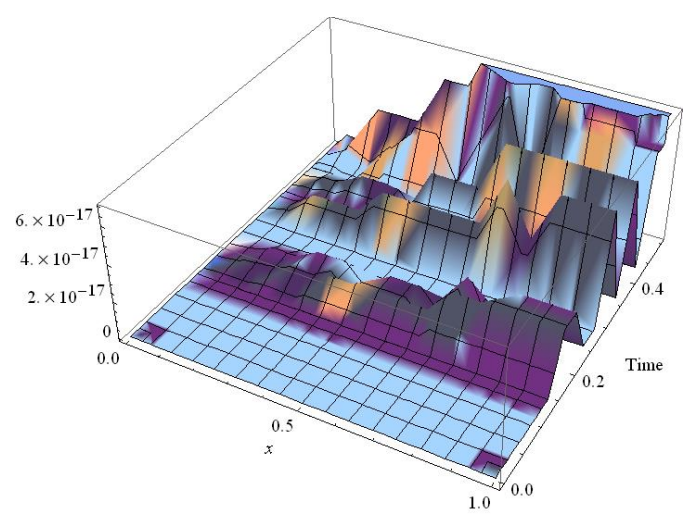

Fig. 1. Absolute errors for sixth order OPIM solution for Example 1.

Example 2) Consider the Telegraph equation [18]

$$
u_{t t}+6 u_{t}=9 u_{x x}
$$


under the initial conditions

$$
u(x, 0)=\sin x, \quad u_{t}(x, 0)=0 .
$$

An initial function can be taken as

$$
u_{0}=\sin x
$$

Using the Eqs. (10),(12),(29) with initial conditions and proceeding as in previous example, we can procure the following approximate solutions:

$$
\begin{gathered}
u_{1}=\sin x-\frac{9 P_{0}}{2} t^{2} \sin x \\
u_{2}=\sin x-\frac{9 P_{0}}{2} t^{2} \sin x+\frac{9}{8} t^{2} \sin x\left(-4+4 P_{0}+8 t P_{0}+3 t^{2} P_{0}\right) P_{1} \\
u_{3}=\sin x-\frac{9 P_{0}}{2} t^{2} \sin x+\frac{9}{8} t^{2} \sin x\left(-4+4 P_{0}+8 t P_{0}+3 t^{2} P_{0}\right) P_{1} \\
-\frac{9}{80} t^{2} \sin x\left(\begin{array}{l}
40-40 P_{0}-80 t P_{0}-30 t^{2} P_{0}-40 P_{1}-80 t P_{1}-30 t^{2} P_{1}+40 P_{0} P_{1} \\
+160 t P_{0} P_{1}+180 t^{2} P_{0} P_{1}+72 t^{3} P_{0} P_{1}+9 t^{4} P_{0} P_{1}
\end{array}\right) P_{2}
\end{gathered}
$$

and so on. Corresponding residual will be in the form:

$$
\begin{aligned}
& \operatorname{Re}\left(x, t ; P_{0}, P_{1}, P_{2}\right)=9\left(u_{3}\right)_{x x}-\left(u_{3}\right)_{t t}-6\left(u_{3}\right)_{t}=\frac{9}{80} \sin [x] \times \\
& -10\left(-4\left(-2+\left(2+12 t+9 t^{2}\right) P_{2}\right)+P_{1}\left(\begin{array}{l}
-4\left(2+12 t+9 t^{2}\right) \\
+\left(8+96 t+216 t^{2}+144 t^{3}+27 t^{4}\right) P_{2}
\end{array}\right)\right)+ \\
& P_{0}\left(\begin{array}{l}
\left.-10\left(\begin{array}{l}
-4\left(2+12 t+9 t^{2}\right) \\
+\left(8+96 t+216 t^{2}+144 t^{3}+27 t^{4}\right) P_{2}
\end{array}\right)+P_{1}\left(\begin{array}{l}
-10\left(8+96 t+216 t^{2}+144 t^{3}+27 t^{4}\right) \\
+\left(\begin{array}{l}
80+1440 t+5400 t^{2}+7200 t^{3}+ \\
4050 t^{4}+972 t^{5}+81 t^{6}
\end{array}\right)
\end{array}\right)\right) \\
\left.\left(\begin{array}{l}
2 \\
4
\end{array}\right)\right)
\end{array}\right.
\end{aligned}
$$

for the third order OPIM approximate solution. With the help of equations (15) and (35), one can reach the following values

$$
P_{0}=0.9022357, P_{1}=-1.0536142, P_{2}=0.5056331
$$

Furnishing (36) into (34), third order OPIM solution arises as:

$$
\begin{aligned}
& u_{3}=\sin x-4.06006065 t^{2} \sin x-1.185315975 t^{2}\left(-0.3910572+7.2178856 t+2.7067071 t^{2}\right) \sin x \\
& -0.0568837 t^{2} \sin x\left(8.03080618-139.98705 t-166.56814714 t^{2}-68.443800 t^{3}-8.555475 t^{4}\right)
\end{aligned}
$$


If the iteration continues, more approximate solutions can be reached. Fig. 2 shows absolute errors for approximate results in the 6 th order.

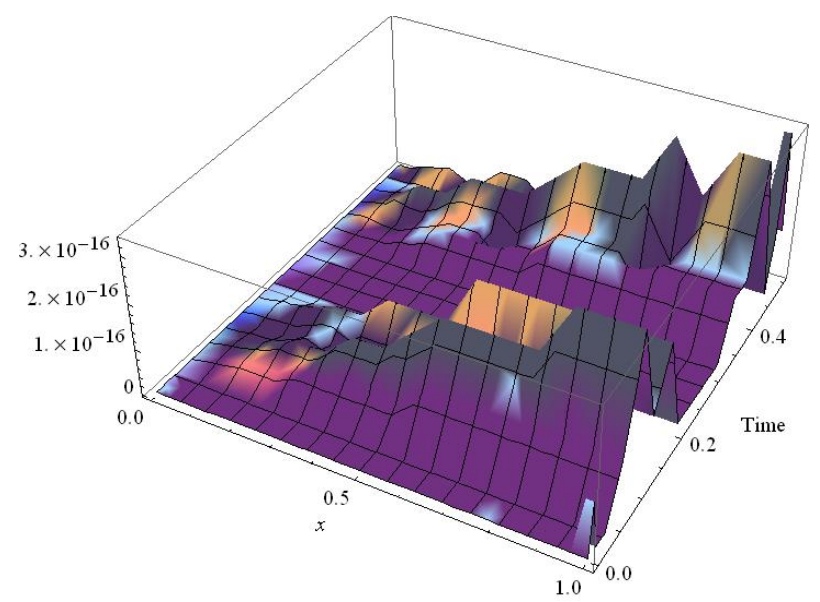

Fig. 2. Absolute errors for sixth order OPIM solution for Example 2.

\section{Conclusion}

In this research, we apply the newly found technique, OPIM, for solving the telegraph type partial differential equations. Two applications are provided to show the effectiveness of the method. Figures prove the accuracy in higher order approximate solutions. All complex computations are handled by Mathematica 9.0. due to huge amount of calculations. We can finally say that in the light of data in this paper, this method can be extended to solve all types of ordinary and partial differential equations.

\section{References}

[1] Baskonus, H. M., \& Hasan, B. (2016). Exponential prototype structures for $(2+1)$-dimensional Boiti-Leon-Pempinelli systems in mathematical physics. Waves in Random and Complex Media, 26(2), 189-196.

[2] Bildik, N., \& Sinan, D. (2015). Implementation of taylor collocation and adomian decomposition method for systems of ordinary differential equations. AIP Conference Proceedings.

[3] Bildik, N., \& Sinan, D. (2013). Applications of Taylor collocation method and Lambert W function to systems of delay differential equations. TJMCS, 13.

[4] Demiray, S. T., Yusuf, P., \& Hasan, B. (2016). All exact travelling wave solutions of Hirota equation and Hirota-Maccari system. Optik-International Journal for Light and Electron Optics, 127(4), 1848-1859.

[5] Bildik, N., and S. Deniz. (2015). Comparison of solutions of systems of delay differential equations using Taylor collocation method, Lambert W function and variational iteration method. Scientia Iranica. Transaction D, Computer Science \& Engineering, Electrical, 22(3), 1052.

[6] Bildik, N., \& Sinan, D. (2015). Modified Adomian decomposition method for solving Riccati differential equations. Review of the Air Force Academy, 3, 21.

[7] Bildik, N., \& Sinan, D. (2015). On the asymptotic stability of some particular differential equations. International Journal of Applied Physics and Mathematics, 5(4), 252.

[8] Grossmann, F., \& Werner, K. (2009). A finite-difference implementation of the Caldeira-Leggett master equation. The Journal of Chemical Physics, 130(3), 034105.

[9] Bildik, N., \& Sinan, D. (2016). The use of Sumudu decomposition method for solving predator-prey systems. Mathematical Sciences Letters, 5(3), 285-289. 
[10] Deniz, S., \& Necdet, B. (2016). Application of adomian decomposition method for singularly perturbed fourth order boundary value problems. AIP Conference Proceedings. Eds. Theodore Simos, and Charalambos Tsitouras.

[11] Gilbarg, D., \& Neil, S. T. (2015). Elliptic Partial Differential Equations of Second Order. Springer.

[12] Ames, W. F. (2014). Numerical Methods for Partial Differential Equations. Academic Press.

[13] Taylor, M. (2013). Partial differential equations II: Qualitative studies of linear equations. Springer Science \& Business Media.

[14] Freidlin, M. I. (2016). Functional Integration and Partial Differential Equations. Princeton University Press.

[15] Fox, L. (2014). Numerical solution of ordinary and partial differential equations: Based on a summer school held in Oxford. August-September 1961. Elsevier.

[16] Atangana, A. (2015). On the stability and convergence of the time-fractional variable order telegraph equation. Journal of Computational Physics, 293, 104-114.

[17] Goldstein, S. (1951). On diffusion by discontinuous movements, and on the telegraph equation. The Quarterly Journal of Mechanics and Applied Mathematics, 4(2), 129-156.

[18] Deniz, S., \& Necdet, B. (2014). Comparison of adomian decomposition method and Taylor matrix method in solving different kinds of partial differential equations. International Journal of Modeling and Optimization, 4(4), 292.

[19] Bildik, N., \& Sinan, D. (2017). Modification of perturbation-iteration method to solve different types of nonlinear differential equations. AIP Conference Proceedings. AIP Publishing.

[20] Bildik, N., \& Sinan, D. (2016). Comparative study between Optimal Homotopy Asymptotic Method and Perturbation-Iteration Technique for different types of nonlinear equations. Iranian Journal of Science and Technology, Transactions A: Science, 1-8.

[21] Bildik, N., \& Sinan, D. (2017). A new efficient method for solving delay differential equations and a comparison with other methods. The European Physical Journal Plus, 132(1), 51.

[22] Pakdemirli, M. (2013). Review of the new perturbation-iteration method. Mathematical and Computational Applications, 18(3), 139-151.

[23] Deniz, S., \& Necdet, B. (2016). Optimal perturbation iteration method for Bratu-type problems. Journal of King Saud University-Science.

[24] Deniz, S., \& Necdet, B. (2017). Applications of optimal perturbation iteration method for solving nonlinear differential equations. AIP Conference Proceedings.

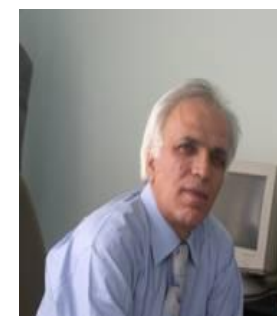

Necdet Bildik was born in Sivas, Turkey in 1951. He graduated from Ankara University in 1974. He earned the M.Sc. degree in University of Louisville, Kentucky, USA in 1978. He awarded the Ph.D. degree in Oklahoma State University, USA in 1982. He was assistant professor in 1988 and also he was became associate professor in 1995. He was promoted to be professor in 2003. He is interested in numerical analysis, ordinary, partial and non-linear differential equations, ergodic theory, stability theory.

He has over than a hundred published articles in the national and international journals and conferences. He also serves as a reviewer for many international journals. 